\title{
Review and Analysis of a Proposed Concept for Energy Efficiency on UWB-MAC in Manet with Directional Antenna
}

\author{
Murtala Muhammad \\ University of Salford, Manchester \\ School of Computing, Science and \\ Engineering \\ M5 4WT
}

\author{
Martin Hope \\ University of Salford, Manchester \\ School of Computing, Science and \\ Engineering \\ M5 4WT
}

\author{
Abubakar Gidado Halilu \\ University of Salford, Manchester \\ School of Computing, Science and \\ Engineering \\ M5 4WT
}

\begin{abstract}
Energy efficiency is a key factor that determines the lifetime of a MANET, and antennas play a vital role in achieving the successful transmission of information. Because nodes in a MANET are normally battery powered, antenna and transmission efficiency is paramount when transmitting information from a node to another. Historically, it has been shown that the use of directional antennas in wireless networks can conserve energy due to the skewed transmission towards a specific direction, and also help to resolve issues of interference from nodes in other directions. However, several concepts and algorithms have emerged that attempt to overcome the irregularities encountered in the use of directional antennas; especially from the design and implementation perspective. This work clearly demonstrates a vivid definition of an UWB MAC protocol for MANETs, and its behaviour when running different applications. It brings to light the valued significance in the implementation of an UWB MAC in a MANET and overcomes the main issues listed in a summary of existing work. The key aim of this research is to design an improved MAC protocol for UWB systems, in order to minimise power consumption in a MANET. Using a simulated environment modelled on the IEEE 802.15.3 standard, key system design parameters for UWB MAC were identified and tested using directional antennas. As a result of this work, a proof of concept improvement via the modification of an existing MAC protocol based on the Ultra WideBand Concept for Ad Hoc Networks (UCAN) using directional antenna techniques is presented. The final results of the analysis clearly show that the proposed UWB MAC performs better when compared to existing MAC protocols.
\end{abstract}

\section{Keywords}

MANET, UWB-MAC, Energy Efficiency, Directional Antenn $a$

\section{INTRODUCTION}

Energy inefficiency in Mobile Ad hoc Network is a major concern in the world of wireless sensor networks which becomes a concern for researchers to intervene. Considering the three different generations of MANETs which have been explored in different research articles and how the generations were implemented and used, the achievements came through strategic design approaches that led to successful advancement in the field. The design of MANETs incorporates the fundamental architecture of general network structure which is referred to as the Open System Interconnect (OSI) model. In designing MANETs, many challenges arise, beginning with the physical to the application layer. This paper aimed to explore a systematic review and analysis on the propsed concept to optimise energy through UWB-MAC in MANETs. Three widespread problems characterise the design challenges: cross-layer interaction, mobility and scaling issues as stated in [1]. The most challenging issue in cross-layer interaction design is to keep all the layers of the network communicating efficiently and collaboratively. However, some of the algorithms designed may not be feasible due to the nature of MANETs in terms of the dynamically changing situation. Mobility is the second challenging issue when designing MANETs and distinguishes MANETs from other networks. The third issue in MANET design is the scaling issue and this constitutes a major problem because of the growing number of nodes in the network. As the number of nodes increases, so too does the complexity[2], especially when the mobility increases. The applications of an ad hoc network are unique because of its design requirements. These design requirements include selfconfiguration, mobility, dynamic topology and multicasting[3]. Most of its applications rely on the existing circumstance that leads to the establishment of the network. Examples can be found in medical responses to tragedy, crime responses to insurgency and fire outbreaks. All these can be established and maintained for both data and real time traffic.

The paper is organised as follows after introduction. The second section of the paper described the sources for energy inefficiency in MANETs followed by analysing the existing MAC protocols in MANETs. As UWB is the key tool in the paper for the optimisation, the paper described some parametric variables and matrices to be consider while designing a MAC protocol. Section six is the summary of review and followed by some assumptions for actualisation of the protocol. Results were presented after preliminary testing of the protocol and performance evaluation is presented as comparative analysis between existing protocols and proposed concept. Finally summary of analysis is presented followed by conclusion.

\section{SOURCES FOR ENERGY INEFFICIENCY IN MANET}

To come up with power parameters to be used for the research implementation, the causes of energy inefficiency in MANET have to be considered, with a brief explanation given in order to justify the need for the parameters. The most common sources of energy inefficiency in MANET are collision, overhearing, over emitting, idle listening and protocol overhead[4].

Collision is consider as the first factor as a source for energy in-efficiency in this paper. Collision occurs in a network as a result of traffic mash in the communication channel. This can be as a result of two or more devices want to access the 
medium at the same time. Collision can also occur as a result of hidden and exposed terminals in a network, especially in a distributed network[5]. Also, if a network is over populated as a result of many terminals participating, the rate of collision will be high, especially when using techniques that cannot avoid or detect collision. Collision is one of the sources of energy inefficiency in MANET and this can be addressed by developing a powerful UWB-MAC protocol. Whilst many techniques are in place for avoiding and detecting collisions in a network like CSMA/CD and CSMA/CA, an enhanced MAC protocol is still needed for optimisation.

The second is overhearing which occurs in a network when other devices capture information that is not destined for them. This occurs as a result of exposed terminals, where terminals listen to information broadcast by other terminals in order to know the destination of that information, to avoid transmission while the terminal is also transmitting. If there is a great deal of overhearing in a network, the energy consumption of the terminals will be high. There are different techniques in place to overcome the problem of overhearing in MANET, especially at the MAC layer, but a lot still needs to be done.

Another factor is over emitting which occurs when a particular node sends information to an unprepared terminal[6]. This can occur when the terminal is out of range, is in a sleep mode, in an idle mode or when the terminal is busy. All these features will consume energy in one way or another.

Other researchers consider idle listening as a factor too which is the time when a node waits for the next incoming transmission[7]. The node is active and energy is utilised. The energy consumed while the terminal is in idle listening serves as wasted energy, thereby causing the terminal to lose much of its energy.

Protocol overhead is another factor that occurs when excess bandwidth is used to send information about other terminals and their routing information[8]. The energy wasted for gathering information about other devices will have effects on the bandwidth allocated to a particular terminal and this will cause low throughput in the network, resulting in low quality of service.

\section{MAC PROTOCOLS ANALYSIS}

In this section, the focus will only be on sensor MAC protocols because of the broadness of the protocol. Medium Access Control (MAC) is mainly classified into two main groups and also according to different approaches used in managing the protocol: contention based and schedule based MAC protocols [9]. Contention based MAC is distributed like protocols which do not require the central co-ordinating node to support transmission or reception in the medium[10]. There are different contention base MAC protocols that are used in wireless sensor networks, for example, Sensor MAC (SMAC), Berkeley Media Access control for low-power sensor Networks (B-MAC) and Predictive Wake-UP MAC (PWMAC) [11].

These MAC protocols operate in different modes, for example, the S-MAC mode of operation is by placing an active node in a state that listens to the medium and has the advantage of a sleeping schedule which drastically reduces energy consumption in the medium. Another advantage of using S-MAC is its easy adaptation to frequent topology changes in the medium and also, it does not require an additional device to aid communication in the medium. Figure
1 shows S-MAC in full operation with four participating devices. The major disadvantage of S-MAC is that it lacks the need to maintain synchronisation for the communication to execute properly[10].

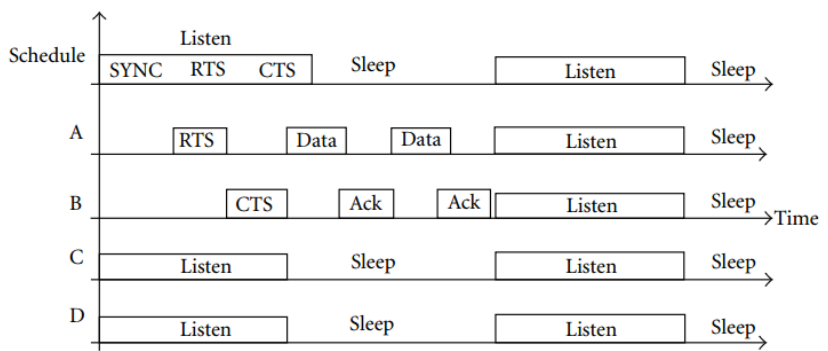

Figure 1: S-MAC protocol operation[9]

Other disadvantages of S-MAC include clock drift which sometimes has to do with nodes participating in the medium and normally affects the performance of the medium resulting in nodes becoming unsynchronised. Also, the occurrence of idle listening in the medium affects it, and the control frames used to generate overhead increase energy usage in the medium. In view of these setbacks, the protocol was reviewed and different protocols were developed using the same techniques with improved performance. Examples of these are dynamic sensor MAC (DS-MAC) and timeout MAC (TMAC). Still more enhancements were suggested and this gave birth to the B-MAC protocol with a different approach that reduced the problems in S-MAC.

The Berkeley Media Access Control for Low-Power Sensor network (B-MAC) is a distributed protocol that was designed to reduce idle listening to save energy usage in many protocols. The protocol uses a back-off time technique which forces the nodes to wait until the channel is clear before sending a packet to the medium. Some refer to the protocol as Low-Power-Listening (LPL) which always checks the channel periodically before sending and receiving data for transmission; this saves much energy in the medium. Figure 2 demonstrates how B-MAC operates.

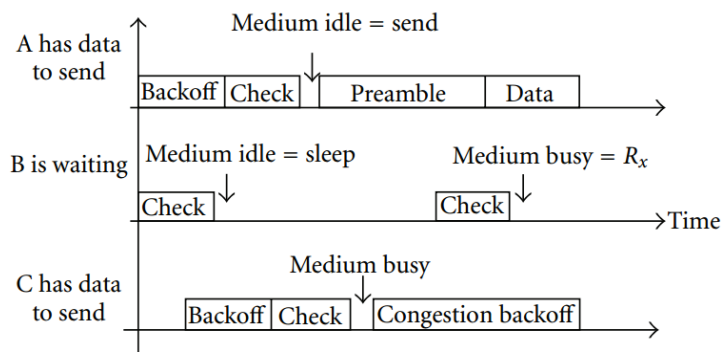

Figure 2: B-MAC operation example[9]

The full operation details of B-MAC protocol are in [9]. Part of the advantage of using the B-MAC protocol is that the protocol is hardware base implemented and it does not require RTS, CTS, ACK or a control frame to support processes in the medium. Also, the protocol does not require synchronisation but it has easy adaption to higher layer stacks for the support of different applications. The only disadvantage of the protocol is that its preamble creates a large overhead which affects the protocol performance. For example, it uses a preamble of 271 bytes to send 36 bytes of data to a channel [9].

The final protocol under contention based MAC is Predictive Wake-Up MAC (PW-MAC) [12]. The protocol is more like a hybrid protocol that combines the features of S-MAC and B- 
MAC because it uses pseudo-random schedules which allow many to transmit at the same time while still avoiding a collision in the medium. The detailed operations of the protocol are contained in [12] and also some limitations of the protocol. The major advantages of PW-MAC are that the protocol can be tested and implemented in hardware using MicaZ motes, the memory footprint of the protocol is small and the protocol sleeps until the receiver is up which in turn, reduces the duty life cycle.

The second category of MAC protocols is schedule based MACs which divide the medium into time slots and in an orderly form for stations to transmit, receive or be inactive. The beauty of the protocol is that each node has a specific time for transmission and after that, the node becomes inactive so as to save energy for the rest of the time. Examples of schedule based MACs are Power-Efficient and Delay-Aware MAC (PEDAMACS), Priority-Based MAC (PRIMA) and Low-Energy Adaptive Clustering Hierarchy (LEACH). The operational details of these protocols can be found in [9]. Table 1 summarises some of the wireless sensor network MAC protocols based on their names, implementation strategy, application, synchronisation requirement and overhead

Table 1: Summary of wireless sensor networks MAC protocols

\begin{tabular}{|l|l|l|l|l|}
\hline MAC & $\begin{array}{l}\text { Implementation } \\
\text { process }\end{array}$ & Application support & $\begin{array}{l}\text { Synchronisation } \\
\text { requirement }\end{array}$ & Overhead \\
\hline S-MAC & Hardware & $\begin{array}{l}\text { Event-driven, long idle periods, } \\
\text { delay order of message time }\end{array}$ & Loose & RTS, CTS, ACK, SYNC \\
\hline B-MAC & Simulation/hardware & Delay tolerant & None & Preamble \\
\hline PW-MAC & Hardware & Low delay, long idle periods & None & Beacon \\
\hline LEACH & Simulation & $\begin{array}{l}\text { Periodic data collection and } \\
\text { monitoring }\end{array}$ & Tight & ADV, join-Req, schedule \\
\hline PEDAMACa & Simulation & Delay bounded & Tight & $\begin{array}{l}\text { RTS,CTS,ACK, } \\
\text { Topology learning }\end{array}$ \\
\hline IEEE 802.11 & Simulation & Different QoS & $\begin{array}{l}\text { Synch, } \\
\text { election }\end{array}$ \\
\hline IEEE 802.15.1 & Simulation & $\begin{array}{l}\text { High data rates, large energy } \\
\text { source, smart terminals } \\
\text { energy consumption }\end{array}$ & None & RTS, CTS, ACk \\
\hline IEEE 802.15.3 & Simulation & $\begin{array}{l}\text { High data rate and low energy } \\
\text { consumption }\end{array}$ & Tight & Synch transmission, S,C \\
\hline IEEE 802.15.4 & Simulation/hardware & $\begin{array}{l}\text { Medium to low data rate, low } \\
\text { energy consumption }\end{array}$ & Tight & Beacon, Ack \\
\hline WirelessHART & Simulation/hardware & Process automation & Tight & Sync, Schedule, routing \\
\hline ISA100a & Simulation/hardware & Process automation & Sync, Schedule, routing \\
\hline
\end{tabular}

\section{ULTRA WIDEBAND PARAMETERS AND MATRICES}

According to FCC and the Ultra WideBand Concept for Ad hoc Networks (UCAN), the design of a new MAC protocol for the UWB system in MANET should be based on the existing MAC protocol in order to provide interoperability between existing narrowband technology and UWB technology [13]. The design should organize the short range communication, hotspot coverage and network backbone interconnection and finally, clusters of ad hoc networks with high density of nodes considering positioning/localisation using manage hopping [14]. Therefore, the architecture of this proposed UWB MAC would be based on UCAN architecture with respect to Wireless Personal Area Network (WPAN) and medium (WLAN) range but specifically on power consumption.

In [14], the paper suggest that the UWB-MAC protocol must have the following attributes, especially when trying to minimise power consumption, that is, high channel acquisition time as a result of long synchronisation, ranging abilities, low power operation and carrier-less pulse position modulation. UWB design parameters that are required in a wireless network can be determined by the method in which a user can decode a specific data stream, giving priority to all users to access the medium of transmission and alter the design architecture of the existing techniques to provide sufficient performance in the communication system [14]. In this research study, the focus will be on redesigning the existing architecture of the MAC protocol in MANET in order to provide sufficient performance in terms of power efficiency. The proposed MAC would have the unique features of UWB systems.

Adopting the UCAN approach of designing the proposed MAC protocol and coming up with the power parameters is the best approach so far. This is because it will solve the problem of localisation with respect to ranging, distance measurement and also part of routing when considering crosslayer design [14]. The general assumption made by UCAN for associating UWB systems, especially when designing MAC protocols for MANET, is based on a link additive cost function. The summation of cost effectiveness of power consumption, network setup, interference in terms of different modulation techniques, quality of communication and the total delay are such that be summarise as in equation 1 .

Power:

$C=C($ PowerConsumption $)+C($ NetworkSetup $)+$ $C($ Interference $)+C($ QualityO Service $)+$ C(TotalDelay) 


\section{Equation 1: General UCAN Equation}

\section{Where $C(x, y)$ is the Cost Function}

However, considering that the research focuses only on power optimisation for the time being, the idle power parameters should therefore be:

$C($ Power $)=C_{1} * R(x, y) * d^{\propto}(x, y)$

\section{Equation 2: Power Equation}

Where:

$\mathrm{C}_{1}=$ is a constant value to be changed for bit rate

$\mathrm{R}=$ is the request rate

$\mathrm{d}=$ is the distance between the two terminals and

$\alpha=$ is the positive number based on propagation characteristic (usually between $2-4$ )

Equation 2 can be implemented using [15] approach of transmitting power, bit rate, choice of modulation technique and ignoring the effect of interference in the communication model. Below is the full detail of the techniques in terms of transmission:

- Interference with respect to different modulation techniques: transmit even outside region regardless of interference

- $\quad$ Bit rate allocation: bit rate is at range of $1-18 \mathrm{Mbps}$

- Modulation techniques to be used: Pulse Position Modulation (PPM) as UWB signal

- Transmitting power: full power during transmission

- Interference with other narrowband systems: the code channel is capable of adapting the transmission medium by ignoring interference and adjusting to the required bit rate for transmission

Table 2: Spectrum Properties

\begin{tabular}{|l|l|l|}
\hline S/N & Entity & Value \\
\hline 1 & Range & $\begin{array}{l}3.1-10 \mathrm{GHz} \text { and is divided } \\
\text { into } 11 \text { channels }\end{array}$ \\
\hline 2 & Bandwidth & $499.2 \mathrm{MHz}$ for each channel \\
\hline 3 & PHY specification & IR \\
\hline 4 & Modulation Scheme & $\begin{array}{l}\text { either on-off keying (OOK) } \\
\text { or Different Binary Phase } \\
\text { Shift Keying (DBPSK) }\end{array}$ \\
\hline
\end{tabular}

\section{Mobility:}

Mobility is another challenging factor after high energy consumption by the mobile nodes in MANET. The proposed MAC protocol will also address the issue of mobility because of the dynamic nature of MANET. Designing a MAC protocol based on the above approach will also solve part of the mobility issues in MANET without considering a specific technique that will solve mobility.

\section{Throughput:}

As the research focused towards using the wireless Personal Area Network (PAN) protocol of said IEEE 802.15.3 for optimisation with regard to power saving, throughput optimisation also has to be regarded to help achieve the desired goal. [15] approach does not consider throughput as an entity for implementation but, if supplemented, it may serve as an added advantage. The following entities have to be

\section{Signal Transmission:}

With respect to signal transmission, [15] approach is also adequate to determine the exact signal transmission where the total time (T) taken for the transmission, chips (c) and short duration time in nano seconds $\left(\mathrm{T}_{\mathrm{c}}\right)$ can be calculated as stated below in the equation 3 :

Time $(T)=\frac{\operatorname{Chips}(\mathrm{c})}{\text { Short duration }\left(\text { in Nano Seconds }\left(T_{c}\right)\right)}$

\section{Equation 3: Signal Transmission Equation}

However, the total time taken for the whole transmission can be presented in the form of frames.

The overall transmission can be determined by the use of a random number generator which can be in the form of the below equation 4 .

$x_{n+1}=\left[a x_{n}\right](M o d m)$

\section{Equation 4: Random Number Generator}

To achieve maximum utilisation of power in the transmission in order to optimise the performance of MAC, the frame size, or PRP, must be large. [15] suggested the PRP of 280 which can be increased or decreased depending on the size of the network.

\section{Modulation:}

With regards to modulation techniques to be used for the proposed MAC, UWB transmission supports both impulse radio (IR) and multi carrier techniques[16]. The research focuses on using Impulse Radio (IR-UWB) transmission to design the UWB-MAC protocol in MANET for optimum power consumption. The reason for using IR-UWB is because it consumes less energy compared to MC-UWB. IR-UWB has unique properties like rise time, pulse width and Pulse Repetition Frequency (PRF)[17]. In addition, IR-UWB is short range, supports low complexity hardware and consumes less power. The proposed UWB-MAC MANET would be based on the spectrum properties in Table 2 .

controlled in order to achieve maximum throughput in MANET for the proposed MAC protocol:
i. Signal-to-Noise Ratio (SNR)
ii. Bandwidth availability
iii. Hardware limitation
iv. Network Allocation Vector (NAV)

The above entities can be measured directly using the Time Window $\left(\mathrm{T}_{\mathrm{w}}\right)$ approach.

[15] approach considered the first three entities which are SNR, bandwidth availability and hardware limitation. Our proposed MAC protocol will also incorporate the use of the NAV entity in order to boost the performance of the protocol and yield high throughput for better quality of service. NAV allocation in designing a MAC protocol can either be RTS/CTS/DATA techniques or allocating numerical values to the frame header. RTS/CTS/DATA technique is a virtual way of allocating NAV while the numerical method is physical. Both methods support auto update which is accepted during implementation.

Finally, the proposed MAC will constitute the characteristics below: 
- $\quad$ Adapt to the varying channel

- $\quad$ Support medium mobility level

- $\quad$ Suitability for UWB and MANET

- Simple design as compared to other approaches in terms of power utilisation as if there is no power control used

In summary, energy can be optimised in the proposed MANET UWB-MAC through the following steps:

i. Maximum power can be used at the transmission stage instead of the receiving stage

ii. Ignoring interference outside the exclusive region

iii. Finally, the exclusive region will always be in a temporal mode

\section{UWB TRANSMISSION TECHNIQUES AT THE MAC LAYER WITH DIRECTIONAL ANTENNA}

The existence of power saving techniques at the MAC layer is a vital issue due to the fact that it constitutes one of the basic layers at which initialisation of communication is instigated. However, among the contemporary techniques are sleeping scheduling techniques, awareness of battery usage at the MAC layer, which is sometimes referred to as the Battery Aware MAC protocol (BAMAC). Different transmission techniques are present at the MAC layers using UWB systems due to their momentous advantage to save energy in the system. The technique includes using Time-hopping Impulse Radio (THIR) [18] in conjunction with directional antenna systems for the improvisation of energy in WSN.

The advantages of using directional antennas in MANET instead of the default antenna system, that is, the omnidirectional system, are enormous. However, Figure 3 is an example which illustrates the motivation behind using the directional system.

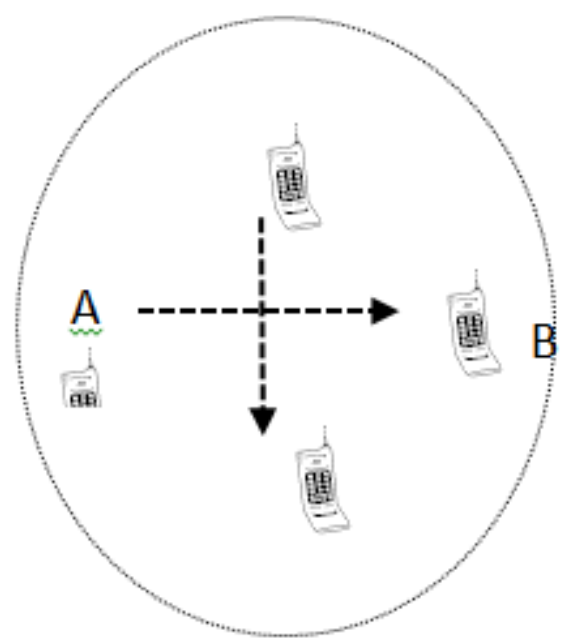

i. Omni Directional mode

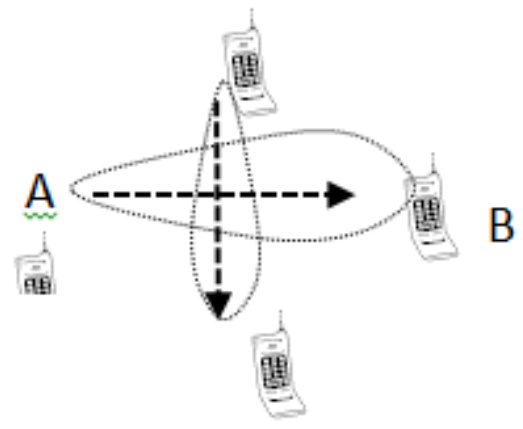

ii. Directional mode.

Figure 3(i),(ii): Omni and Directional System

Some of the advantages are spatial reuse factor, energy saving and extending range in terms of location finding which are explained in detail below.

i. Energy saving with directional antennas: In Figure 3(ii), communication between nodes A/B and $\mathrm{D} / \mathrm{C}$ will strengthen the flow of communication even when the signal is weak due to the directivity of the transmission and the high reception gain of the antenna. Therefore, energy required for the nodes to communicate is less, unlike in Figure 3(i) which uses the Omni system. In addition, it will reduce the power required for communication with a maximum distance $d$ of communication covered due to the high gain of antenna use [19]. Consequently, the use of directional antenna in MANET and adapting the use of minimum power in transmission reception will save energy significantly.

ii. Spatial reuse of the channel: The bandwidth utilisation in Figure 3(ii) and Figure 3(i) cannot be the same. Nodes in Figure 3(ii) utilise entirely its bandwidth while communicating due to the directivity and the beam will be focused from sender to receiver instead of spreading it. In Figure 3(i), interference may occur due to the Omni system used. Multiple transmission will also occur in (ii) between the nodes because of the directivity, unlike in the Omni system where it must be single transmission to avoid collision. These features of using directional antennae in MANET will increase the spatial reuse factor in the system.

iii. Extending range of communication: As the beam is focusing towards a specific direction in (ii), so also the range of communication increases unlike in (i) where the beam was unfocused. Focused beams travelled a larger distance compared to unfocused beams [18]. In [20], the research quantified that when using directional antennas, it requires less energy/power to reach a maximum distance than when using omni- directional antennas.

With the enormous advantages of using directional antennas, it is quite motivating to implement the use of the system in MANET and other networking setups like Body Area Network and the like. 


\section{SUMMARY OF REVIEW}

In summary, reviews have been undertaken on general aspect of the study in specific consideration on sources for energy inefficiency in MANETs and some existing protocols in MANETs. It has been observed that centralised MAC protocols are best suitable for conserving power in MANET due to their control mode of operation. Also from the different power management techniques reviewed in this study, it is observed that power conservation techniques in MANET are generally implemented at different levels; at the MAC layer, by tuning transmit power or by using optimised power with full UWB technology.

Applying power management technique at the MAC layer is often done by carefully sending the receiver into a sleep mode, or by using a transmitter with adaptable output power and selecting routes that require many short hops, instead of a few longer hops. Furthermore, applying power management technique at node levels reduced energy consumption but to the best of the author's knowledge, power is consumed when mobile nodes are in sleep mode and also while trying to wake from sleep. More so, applying power management technique by controlling transmit power can be quite complicated because of the fact that choice of power level can affect other performances in MANET such as delay, throughput, increase in interference and so on.

Based on the fact that most researches reviewed were based on network with narrow band technology which can be quite complicated in complex networks in terms of power transmission and data transmission when compared to using UWB technology, this paper focus on power conservation technique in a network with full consideration on UWB standard.

Before testing the proposed concept, the paper analysis some protocols and define some basic parameters for consideration while implementing the protocol. Also, UWB transmission techniques at the MAC layer with solid consideration of using directional antenna has been established.

\section{BASIC ASSUMPTION FOR SETTING THE PROPOSED CONCEPT}

This paper uses analytical and simulation methods in the implementation of the study. The study assumed that all mobile nodes are homogenous and may be operated with the same energy level and with the same speed level. Based on that, it is easy to achieve this in a simulation environment and analytical approach, but not in a real life-time scenario because of its complexity and time constraint.

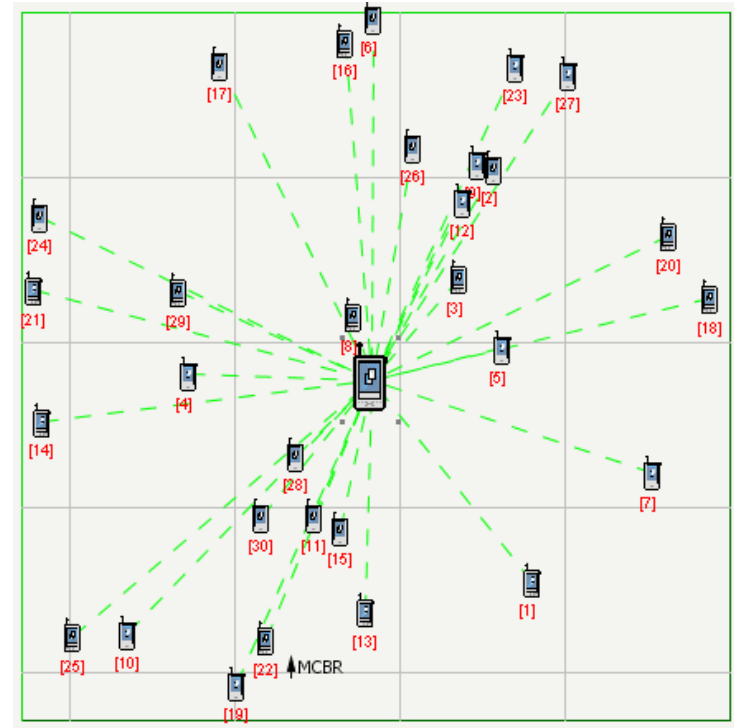

Figure 4: Scenario for Preliminary testing of the proposed mode

\subsection{Preliminary testing of the proposed protocol using QualNet simulator}

Before proposing an analytical model for testing and actualisation of the protocol, the used of a simulation approach to test the proposed model based on some assumptions and some selected parameters with justifications. The analysis was made by creating scenarios based on existing models in QualNet for proper implementation of the proposed protocol.

\subsection{Analysis of the Proposed UWB-MAC with Simulator}

Considering MANET as a multi-hop connection network and the ineffectiveness of using omni-directional antennas as default antennas for transmission and reception purposes, it is assumed that there is a need to come up with a different strategy for transmitting and receiving patterns using directional antennas in order to save energy.

In this research study, the modified model is tested using QualNet Simulator to analyse its outcome using some metrics as described in Tables 3 and 4 . With this analysis, it was expected that potential challenges would be figured out relative to the proposed UWB-MAC and the guidance of future solution paths. The analysis will also demonstrate how the proposed protocol will interact with other layers of the network: specifically, the network layer. Different scenarios are created with the default MAC protocol using the Omni system, the UCAN strategy and the proposed UWB-MAC in order to justify the need for the research study.

Table 3: Simulation Parameters for Omni System

\begin{tabular}{|l|l|l|}
\hline \multicolumn{2}{|l|}{ Omni system } \\
\hline S/N & Parameters & Values \\
\hline 1 & Number of Nodes & 30 \\
\hline 2 & Antenna type & Omni \\
\hline 3. & MAC & IEEE802.15.3 \\
\hline 4 & Antenna range & $200 \mathrm{~ms}$ \\
\hline
\end{tabular}




\begin{tabular}{|l|l|l|}
\hline 5. & Mobility & None \\
\hline 6. & Propagation channel frequency & $9.14 * 10^{8} \mathrm{~Hz}$ \\
\hline 7. & Path loss model & Two ray \\
\hline 8. & Transmission power & $24.5 \mathrm{~dB}$ \\
\hline 9. & Receiver sensitivity & $-68.1 \mathrm{dBm}$ \\
\hline 10. & Routing Protocol & TORA \\
\hline 11. & Transport protocol & TCP \\
\hline
\end{tabular}

Table 4: Simulation Parameters for UCAN approach and Proposed MAC

\begin{tabular}{|l|l|l|}
\hline \multicolumn{2}{|l}{ UCAN and Modified system } \\
\hline S/N & Parameters & Values \\
\hline 1 & Number of Nodes & 30 \\
\hline 2 & Antenna type & Steerable \\
\hline 3. & MAC & IEEE802.15.3 \\
\hline 4 & Antenna range & $200 \mathrm{~ms}$ \\
\hline 5. & Mobility & None \\
\hline 6. & Propagation channel frequency & $9.14^{*} 10^{8} \mathrm{~Hz}$ \\
\hline 7. & Path loss model & Two ray \\
\hline
\end{tabular}

\section{PERFORMANCE EVALUATION}

The paper used a batch experimental mode to run the scenario with multiple parameters in order to obtain results that defined the number of packets dropped, energy consumed at the MAC layer, total battery charge, percentage of time to transmit and the goodput.

One of the responsibilities of a MAC protocol in a network is to make sure that transmission and reception between nodes is successful without collision occurring in the medium, that is, with total avoidance of collision in the network. Whenever collisions occur, packets may drop and communication may halt, re-transmission may occur and the time of transmission may increase which may cause the goodput to drop. In a real time application in an ad hoc network, packets dropped constitute a factor that affects QoS in terms of energy consumption.

Packet drop occurs when packets are transmitted within a time window but not received by the intended receiver. This may occur as a result of traffic patterns, environmental factor and topology.

The analysis is concentrated on the two basic layers, that is, the physical and MAC layers to monitor the number of packets dropped considering the OmniSys, UCAN approach and the proposed UWB-MAC. In Figure 5, the variation of packets dropped at the MAC layers is not great. The used TORA as a hybrid routing protocol to enable the proposed protocol to perform at its best with respect to higher simulation times and for proper adjustment in the routing table. TORA uses both methodologies, that is, active and proactive methods of routing which show that the proposed UWB-MAC performs better. The packets dropped due to simulation time and increasing number of nodes were

\begin{tabular}{|l|l|l|}
\hline 8. & Transmission power & $24.5 \mathrm{~dB}$ \\
\hline 9. & Receiver sensitivity & $-68.1 \mathrm{dBm}$ \\
\hline 10. & Routing Protocol & TORA \\
\hline 11. & Transport protocol & TCP \\
\hline 12. & Direction Gain & $10.0 \mathrm{db}$ \\
\hline 13. & Directional NAV Delta angle & 22.5 degrees \\
\hline
\end{tabular}

Due to QualNet's drawbacks and time constraints, the implementation of the model is based on some assumptions. Three scenarios were created based on OmniSys, UCAN approach and the proposed UWB-MAC and parameters were inserted into the scenarios as in Tables 3 and 4. A total of 30 nodes was considered as the density of the network with an area of $200 \times 200$ metres in an ad hoc environment as shown in Figure 4. Mobility is not an issue given that the existing model covers the issue of mobility in some other papers. The distance between mobile nodes is static and is 10 meters at the furthest.

The directional angle of $22.5^{\circ}$ for the steerable antenna (directional NAV delta angle) was adopted based on the proving analysis conducted in [21]. At that angle, there is less interference due to other frequencies and it is the smallest angle between the edges of the nodes. Analysis was conducted by [21] with respect to the specific angle of transmission in order to yield maximum goodput and different angles were tested.

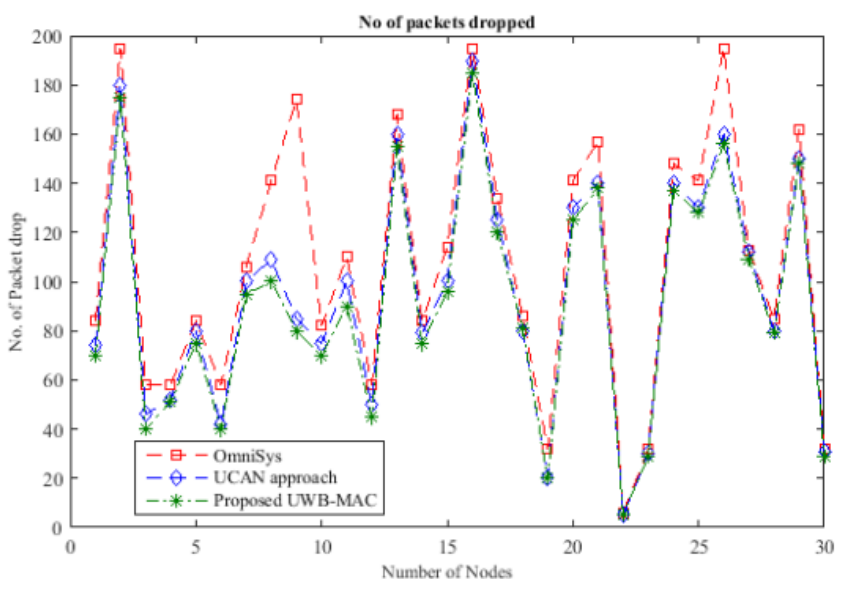

Figure 5: Packet Drop Analysis

consistent in a zig-zag motion. Likewise, variation in frequencies also plays a significant factor in decreasing the amount of packets in the proposed UWB-MAC.

The total packets dropped by OmniSys MAC is $3233 \mathrm{Mbps}$, total packets dropped by UCAN approach is $2855 \mathrm{Mbps}$ and total packets dropped by the proposed UWB-MAC is 2746.5Mbps. 


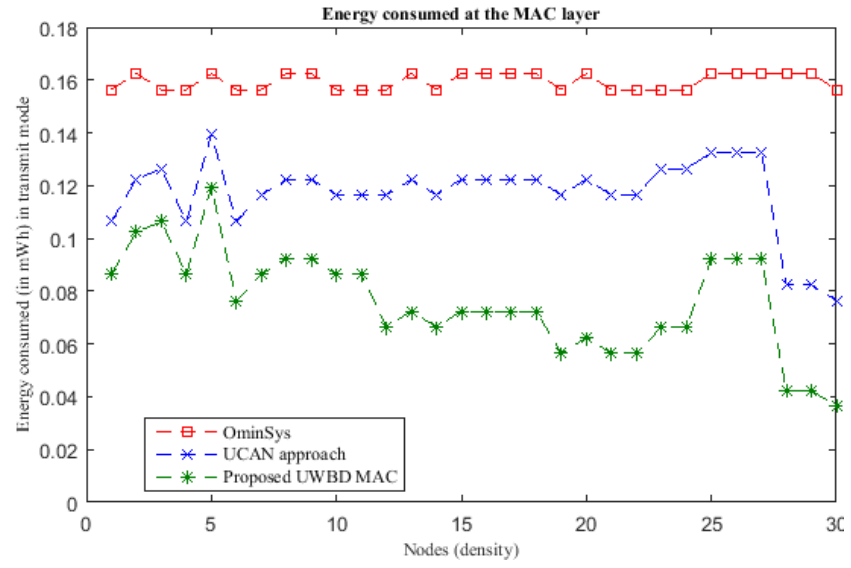

Figures 6 and 7 described the energy consumed at the MAC layer and the total battery charge respectively. The simulation results show that the proposed model conserved energy more than the original model parameters. The battery charge of the nodes rose up to $0.18 \mathrm{mAhr}$ which is high, compared to the UCAN model which demonstrates $0.16 \mathrm{mAhr}$. This saves about $0.02 \mathrm{mAhr}$ and is a significant value to be considered. This recorded increase will save the nodes more life to participate in the network. The proposed UWB-MACs demonstrate more in saving another $0.01 \mathrm{mAhr}$ totalling about
Figure 6: Energy consumed at the MAC Layer

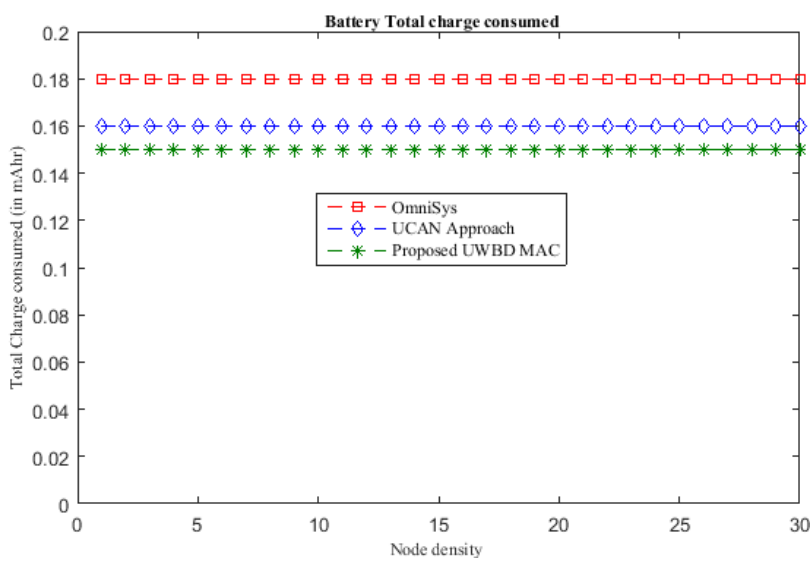

Figure 7: Total Battery Charge Consumed

$0.03 \mathrm{mAhr}$ compared to the original Omni system model. $0.15 \mathrm{mAhr}$ is the code point of the proposed UWB-MAC and this is a significant number to be reflected.

The energy consumed at the MAC layer in continuous transmit mode in Figure 7, with respect to density of the network, was $0.16 \mathrm{mWh}$ downcast to $0.022 \mathrm{mWh}$. The original model recorded a maximum value of $0.162 \mathrm{mWh}$ which is higher than the UCAN model and likewise, higher than the proposed

UWB-MAC.

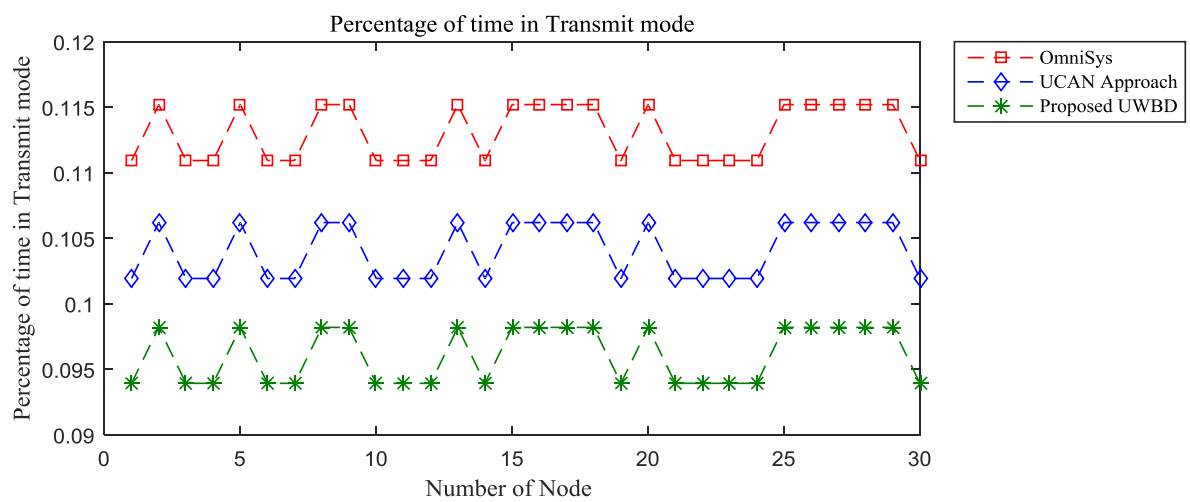

Figure 8: Percentage of Transmitting Time

The percentage of time in continuous transmission mode has to do with time to transmit, receive, idle and sleep of the MAC protocol. All these categories were combined to form a single entity for easy analysis although in some cases, they are independent of each other. With regards to this analysis, it is considered as a single entity because our interest is only in saving energy at the MAC layer without giving emphasis to other layers which may also be considered in the future. The total percentage of continuous time of transmission mode of the proposed UWB-MAC recorded less than the original MAC and the UCAN model.

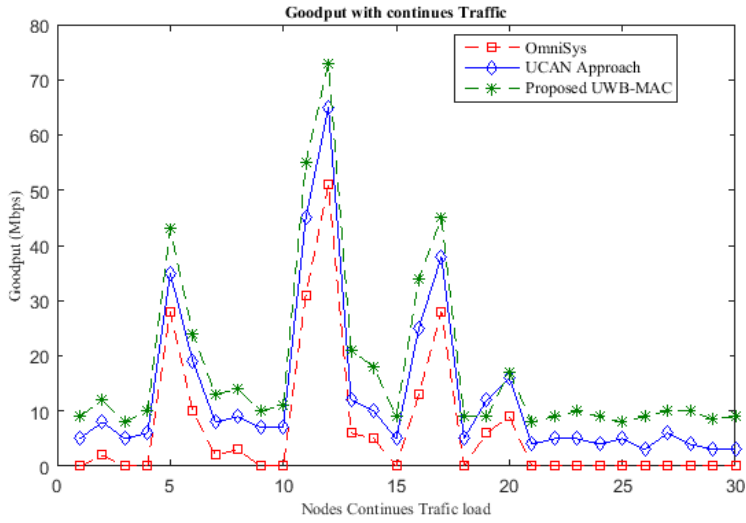

Figure 9: Goodput Comparison 


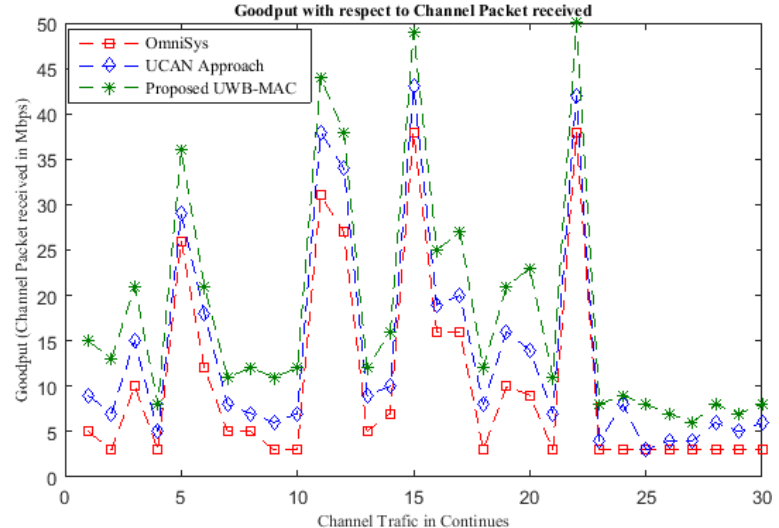

Figure 10: Goodput Comparison

Figures 9 and 10 described the goodput of the network in terms of MAC channel traffic and the traffic load in continuous mode. The three different scenarios were plotted based on the prescribed parameters and the results indicate that the proposed UWB-MAC performed better against the original version and the UCAN approach due to directivity. The goodput of the proposed MAC is a little bit higher than the UCAN approach with a significant increase in number. Moreover, the performance of the MAC protocol, with respect to application, is subject to the lightness of the application. Therefore, heavy application should also be tested. The research predicted that even with the heavy application, it is only the two original protocols that would be affected because of the Omni system in place. The proposed UWB-MAC will survive because of the directivity system in place. The proposed UWB-MAC protocol may have some resulting issues with other layers of the network, especially the immediate layer which is the network stack and thereby, it may also have an effect on the total goodput of the proposed system. Solving these problems will be a task for the future using the analytical approach.

\section{SUMMARY OF ANALYSIS}

The aim of analysing the protocols is to understand the behaviour of the protocols in a real-life situation in terms of communication between nodes and to understand the features of directional systems with UWB properties in MANET. Subsequently, the aim is to pinpoint the sources of inefficiency in order to proffer a solution when developing the analytical model.

The analysis shows that there is significant improvement in goodput and energy consumption both for the battery charge and energy model. The paper concluded that more techniques should be applied at the MAC layer for the effective use of directional antennas. Nevertheless, the study observed that the angle of transmission plays a significant role in utilizing the channel capacity in terms of minimising the transmission time. The study also helps in identifying the full characteristics of directional systems in MANET with UWB properties. Lastly, it is also observed that there is good improvement in our proposed UWB-MAC compared to ordinary omni-directional systems and the UCAN approach. Table 5 exhibits the summary performance of the three protocols.

Table 5: Summary of Analysis

\begin{tabular}{|l|l|l|l|l|l|l|}
\hline $\begin{array}{l}\text { MAC } \\
\text { protocols }\end{array}$ & $\begin{array}{l}\text { No of packet } \\
\text { drooped }\end{array}$ & $\begin{array}{l}\text { Energy consumed } \\
\text { at MAC Laye } \\
(\mathrm{mWh})\end{array}$ & $\begin{array}{l}\text { Total battery } \\
\text { charge consumed } \\
(\mathrm{mAhr})\end{array}$ & $\begin{array}{l}\text { Percentage of time } \\
\text { in transmit mode }\end{array}$ & $\begin{array}{l}\text { Goodput } \\
\text { traffic } \\
(\mathrm{Mbps})\end{array}$ & $\begin{array}{l}\text { Goodput } \\
\text { channel traffic } \\
\text { (Mbps })\end{array}$ \\
\hline OmniSys & 3233 & 4.8 & 5.4 & 3.4 & 194 & 302 \\
\hline $\begin{array}{l}\text { UCAN } \\
\text { approach }\end{array}$ & 2855 & 3.5 & 4.8 & 3.1 & 384 & 411 \\
\hline $\begin{array}{l}\text { Proposed } \\
\text { UWB-MAC }\end{array}$ & 2746.5 & 2.3 & 4.5 & 2.9 & 534.5 & 549 \\
\hline
\end{tabular}

As the research is on-going, it is envisaged that greater benefits of the improved model would be unveiled and articulated. Extended study and analysis of the model capabilities with respect to other areas will be undertaken in a bid to grasp better understanding of the full package of the model. Clearly, further work needs to be done. Table 5 summarises the outcome of the analysis: the proposed UWBMAC performs better compared to the original versions.

\section{CONCLUSION}

This paper analysed protocols to understand the behaviour of the proposed concept in a real life situation towards communicating between nodes and to understand the features of directional systems with UWB properties in MANET. Subsequently, the sources of inefficiency has been define in order to proffer a solution when developing the analytical model. The analysis shows that there is significant improvement in goodput, energy consumption both for the battery charge and energy model. The study concluded that more techniques should be applied at the MAC layer for the effective use of directional antennas. Nevertheless, it is also observed that the angle of transmission plays a significant role in utilizing the channel capacity in terms of minimising the transmission time. The study also helps in identifying the full characteristic of directional system in MANET with UWB properties. The paper also observed that there is good improvement in our proposed UWB-MAC compared to ordinary Omni directional system and UCAN approach. In this research paper, presentation of a design approach for the proposed UWB-MAC using directional antennas and the proposed model constraints. UWB-MAC parameters were established and validated based on traffic model and schematic approach. An analytical model was defined and established, and will be proved via an optimisation approach. The simulation result shows that the proposed UWB-MAC behaved normal compared to the original versions of MAC protocol. 


\section{REFERENCES}

[1] P. Ghosekar, G. Katkar, and P. Ghorpade, "Mobile ad hoc networking: imperatives and challenges," IJCA Special issue on MANETs, vol. 3, pp. 153-158, 2010.

[2] A. Asadi, Q. Wang, and V. Mancuso, "A survey on device-to-device communication in cellular networks," IEEE Communications Surveys \& Tutorials, vol. 16, no. 4, pp. 1801-1819, 2014.

[3] S. Bitam, A. Mellouk, and S. Zeadally, "Bio-inspired routing algorithms survey for vehicular ad hoc networks," IEEE Communications Surveys \& Tutorials, vol. 17, no. 2, pp. 843-867, 2015.

[4] R. Ramya, G. Saravanakumar, and S. Ravi, "MAC protocols for wireless sensor networks," Indian Journal of Science and Technology, vol. 8, no. 34, 2015.

[5] L. Du, L. Chen, A. Yamada, and A. Fujiwara, "Medium access control method and apparatus in wireless distributed network," ed: Google Patents, 2013.

[6] N. Srivastava, S. Seth, M. Kumar, and R. Kumar, "Energy Efficient Protocols for Wireless Sensor Networks: A Review," International Journal of Computer Technology and Applications, vol. 4, no. 3, p. 434, 2013.

[7] B. Jang, J. B. Lim, and M. L. Sichitiu, "An asynchronous scheduled MAC protocol for wireless sensor networks," Computer Networks, vol. 57, no. 1, pp. 85-98, 2013.

[8] M. Altayeb and I. Mahgoub, "A survey of vehicular ad hoc networks routing protocols," International Journal of Innovation and Applied Studies, vol. 3, no. 3, pp. 829846, 2013.

[9] J. Kabara and M. Calle, "MAC protocols used by wireless sensor networks and a general method of performance evaluation," International Journal of Distributed Sensor Networks, 2012.

[10] Y. Wang and J. Zhao, "Multi-objects positioning system and power-control based multiple access control method," ed: Google Patents, 2013.

[11] S. Kaur and L. Mahajan, "Power saving MAC protocols for WSNs and optimization of S-MAC protocol," International Journal of Radio Frequency Identification \& Wireless Sensor Networks, vol. 1, no. 1, pp. 1-8, 2011.
[12] R. Zhang, R. Ruby, J. Pan, L. Cai, and X. Shen, "A hybrid reservation/contention-based MAC for video streaming over wireless networks," IEEE Journal on Selected Areas in Communications, vol. 28, no. 3, 2010.

[13] B. Karaoglu and W. Heinzelman, "A dynamic channel allocation scheme using spectrum sensing for mobile ad hoc networks," in Global Communications Conference (GLOBECOM), 2012 IEEE, 2012, pp. 397-402: IEEE.

[14] A. Gupta and P. Mohapatra, "A survey on ultra wide band medium access control schemes," Computer Networks, vol. 51, no. 11, pp. 2976-2993, 2007.

[15] J.-Y. Le Boudec, R. Merz, B. Radunovic, and J. Widmer, "A MAC protocol for UWB very low power mobile adhoc networks based on dynamic channel coding with interference mitigation," 2004.

[16] S. Bhandari and S. Moh, "A survey of MAC protocols for cognitive radio body area networks," Sensors, vol. 15, no. 4, pp. 9189-9209, 2015.

[17] K. M. S. Thotahewa, J.-M. Redouté, and M. R. Yuce, Ultra wideband wireless body area networks. Springer, 2014.

[18] T. Spyropoulos and C. S. Raghavendra, "Analyzing the Effect of Antenna Type and MAC Protocol on Wireless Ad-Hoc Network Capacity," USC Technical Report2006.

[19] Y.-B. Ko, V. Shankarkumar, and N. H. Vaidya, "Medium access control protocols using directional antennas in ad hoc networks," in INFOCOM 2000. Nineteenth Annual Joint Conference of the IEEE Computer and Communications Societies. Proceedings. IEEE, 2000, vol. 1, pp. 13-21: IEEE.

[20] R. Bhusal, S. K. Timalsina, and S. Moh, "Impact of directional antennas on contention based MAC protocols in ad hoc networks," in Computing and Networking Technology (ICCNT), 2012 8th International Conference on, 2012, pp. 405-408: IEEE.

[21] A. M. Ajofoyinbo and W. W. Li, "Directional antennas MAC protocol for energy-efficient wireless sensor networks with mobile sink," International Journal of Communication Systems, 2015. 